\title{
SBRT for centrally localized NSCLC - What is too central?
}

\author{
J. Roesch ${ }^{{ }^{*}}$ (I), C. Panje ${ }^{1}$, F. Sterzing ${ }^{2}$, F. Mantel ${ }^{3}$, U. Nestle$^{4}$, N. Andratschke ${ }^{1}$ and M. Guckenberger ${ }^{1}$
}

\begin{abstract}
Purpose: Current guidelines recommend stereotactic body radiotherapy (SBRT) for stage I non-small-cell lung cancer (NSCLC) in medically inoperable patients. There are excellent outcome and toxicity data for SBRT of peripheral lung tumors. However, the discussion on SBRT for centrally located tumors is controversial. This study evaluated current clinical practice regarding SBRT of centrally located lung tumors, to identify common fractionation schedules and commonly accepted contraindications for SBRT.

Methods: A questionnaire consisting of two parts was introduced at the annual meeting of the DEGRO working group on stereotactic radiotherapy, representing centers in Germany and Switzerland. The first part of the questionnaire covered general information about the centers, whereas the second part specifically addressed SBRT of centrally located lung tumors, using case examples of nine primary NSCLC patients. Reconstructions of a contrast enhanced $\mathrm{CT}$, as well as PET-Imaging for each case were demonstrated to the participants.

Results: Twenty-six centers participated in the meeting. The majority was academic (73\%), participated in interdisciplinary thoracic oncology tumorboards (88\%) and offered SBRT for lung tumors (96\%). Two centers questioned the indication of SBRT for central lung tumors because of lack of evidence. The majority of centers had experience in SBRT for central lung tumors (88\%) and half of the centers reported more than ten cases treated during a median period of five years. Most fractionation schedules used PTV encompassing doses of 48-60 Gy in eight fractions with maximum doses of 125-150\%.

A clear indication for SBRT treatment was seen by more than $85 \%$ of centers in three of the nine patients in whom tumors were small and not closer than $2 \mathrm{~cm}$ to the main bronchus. Prior pneumonectomy or immediate adjacency to hilar/mediastinal structures were not considered as contraindications for SBRT. In cases where the tumor exceeded $4 \mathrm{~cm}$ in diameter or was located closer than $4 \mathrm{~cm}$ to the carina $50-80 \%$ of centers saw an indication for SBRT. One case, with a $7 \mathrm{~cm}$ tumor reaching to the carina would have been treated with SBRT only by one center.

Conclusion: Within DEGRO working group on stereotactic radiotherapy, SBRT for small $(<4 \mathrm{~cm})$ early stage NSCLC is a common indication, if the minimal distance to the main bronchi is at least $2 \mathrm{~cm}$. The controversy on the treatment of larger and more central tumors will hopefully be solved by ongoing prospective clinical trials.
\end{abstract}

Keywords: SBRT, SABR, NSCLC, Central lung, Pulmonary toxicity

\footnotetext{
* Correspondence: johannes.roesch@usz.ch

'Department of Radiation Oncology, Universitässpital Zürich, Zürich,

Switzerland

Full list of author information is available at the end of the article
} 


\section{Background}

Malignant neoplasms of the lung are the most frequent cause of cancer-related death in the world with approximately 350,000 deaths in Europe and 1.6 million deaths worldwide in 2012 [1-3]. Although the overall prognosis of non-small cell lung cancer (NSCLC) is poor, early stage without regional or metastatic spread can be cured by local treatment. The combination of future screening programs on the basis of computed tomography (CT) and an aging population will most likely increase the incidence of early stage lung cancer, especially in elderly patients $[4,5]$

Surgical lobectomy plus mediastinal lymph node dissection is the standard treatment for early stage NSCLC. However, the increasing number of elderly patients with comorbidities demonstrates the need for less-invasive therapies [6]. Stereotactic body radiotherapy (SBRT) for peripherally located early stage lung cancer has recently emerged as a safe and non-invasive alternative to surgical resection with equivalent rates of local tumor control, and has been established as standard of care for inoperable tumors in specialized centers [7-9].

However, centrally located lung tumors represent a challenge for both surgical treatment and SBRT. Central tumors may more likely require extensive surgery like pneumonectomy, which bears the risk of inferior outcome or inoperability $[10,11]$. Likewise, there is evidence that SBRT of central tumors has an increased risk of severe toxicity, which may be up to 11 times higher than in peripheral tumors [12]. Overall, prospective data on SBRT of central tumors is sparse, but presently recruiting clinical trials will prospectively assess long term benefits and risks of SBRT for this patient population in order to generate solid evidence on local control and toxicity $[13,14]$.

Unfortunately, "central location" is not consistently defined in recent prospective trials, as shown in the following examples: (1) RTOG 0236: a tumor within $2 \mathrm{~cm}$ to the proximal bronchial tree (PBT), which was described as the distal $2 \mathrm{~cm}$ of the trachea, carina, main bronchi and named major lobar bronchi up to their first bifurcation (i.e. upper and lower lobe bronchi, intermedius bronchus, lingular bronchus) $[15,16]$; (2) IASLC recommendation: a tumor within $2 \mathrm{~cm}$ to any mediastinal critical structure, including the bronchial tree, esophagus, heart, major vessels, spinal cord, brachial plexus, phrenic and recurrent laryngeal nerve [17]; (3) RTOG 0813: a tumor within $2 \mathrm{~cm}$ to the PBT or touching the mediastinal pleura [18]; some authors further enlarged the zone by tumors within $6 \mathrm{~mm}$ to the pericardium or $1 \mathrm{~cm}$ around the mediastinum $[19,20]$.
Furthermore, SBRT in daily routine follows not only published evidence but also depends on the physician's experience and expertise, resulting in a variety of treatment schedules. At present, it is unknown whether SBRT can be applied to all centrally located tumors or whether there are locations which are too close to critical organs at risk.

Therefore, this study evaluated the patterns of current clinical practice of SBRT for centrally located lung tumors in 26 German and Swiss centers with the aim to identify common fractionation schedules and commonly accepted contraindications.

\section{Methods}

A questionnaire consisting of two parts was presented to representatives of 26 centers in Germany $(n=25)$ and Switzerland $(n=1)$ during the annual meeting of the DEGRO working group on stereotactic radiotherapy. The first part of the questionnaire addressed general information about the centers such as type (academic vs non-academic), institution size, experience and treatment specifications regarding lung SBRT in general.

In the second part of the questionnaire, the participants were shown nine cases of centrally located primary NSCLC (cT2-4 cN0 cM0) and were asked to determine if SBRT was indicated and which fractionation schedule they would suggest. Furthermore, they were asked to provide reasons if they rejected SBRT in a specific case and to suggest alternative treatments. All presented cases originated from four different academic centers (UniversitätsSpital Zürich, Universitätsklinikum Würzburg, Universitätsklinikum Heidelberg, Klinikum der Universität München) and were consequently anonymized. The survey participants were shown axial, sagittal and coronal reconstructions of a contrast-enhanced 3D$\mathrm{CT}$ of the lungs in combination with fluordeoxyglucose positron emission tomography (FDG-PET) imaging if available. The medical history for all cases was assumed to be identical: a 75-year-old male, active smoker with 25 pack-years and relevant comorbidities of chronic obstructive pulmonary disease $\left(\mathrm{FEV}_{1}=1 \mathrm{l} / \mathrm{s}\right)$, diabetes type 2, hypertension and congestive heart disease after coronary dilatation because of unstable angina pectoris five years ago.

\section{Results}

The majority of the participating centers were academic $(n=19)$, participated in interdisciplinary thoracic oncology tumorboards $(n=23)$ and practiced SBRT for lung tumors as standard $(n=25$, see Table 1$)$. Two centers questioned the suitability of SBRT for central lung tumors because of lack of evidence and therefore did not practice it. The majority of centers had experience in 
Table 1 Individual center characteristics

\begin{tabular}{|c|c|}
\hline \multicolumn{2}{|l|}{ General questionnaire items } \\
\hline Center type & $\begin{array}{l}\text { Academic }=19 \\
\text { Public }=4 \\
\text { Private }=2 \\
\text { Non specified }=1\end{array}$ \\
\hline Median number of linear accelerators & 3 (range $2-7$ ) \\
\hline $\begin{array}{l}\text { Regular participation in thoracic } \\
\text { oncology tumorboard }\end{array}$ & $88 \%$ \\
\hline $\begin{array}{l}\text { Overall experience in SBRT of the } \\
\text { centrally located NSCLC }\end{array}$ & $\begin{array}{l}0 \text { cases }=3 \\
<10 \text { cases }=10 \\
>10 \text { cases }=13\end{array}$ \\
\hline Duration of SBRT experience & 5 years (range: $1-15$ ) \\
\hline \multicolumn{2}{|l|}{ General treatment items } \\
\hline Imaging for target volume definition & $\begin{array}{l}4 \mathrm{D}-\mathrm{CT}=18 \\
\mathrm{PET}-\mathrm{CT}=21\end{array}$ \\
\hline Image guidance & $\begin{array}{l}\mathrm{CBCT}=24 \\
\mathrm{TOMO}=2 \\
\text { Other }=2\end{array}$ \\
\hline Patient immobilization system & $\begin{array}{l}\text { Body Fix/Frame }=11 \\
\text { vacuum matrass }=7 \\
\text { unknown }=7\end{array}$ \\
\hline Abdominal compression & 3 \\
\hline
\end{tabular}

SBRT for central lung tumors. Thirteen centers referred more than ten treated cases during a median period of five years, whereas the remaining ten centers reported less than ten treated cases within a median period of three years.

A FDG-PET scan was an essential component of the staging procedure in each center and, when reasonable, was frequently used for target volume definition ( $n=21,81 \%)$. A 4D-CT scan was standard in $69 \%$ for treatment planning $(n=18)$. Image guidance by cone-beam CT or by another system was practiced by all centers. Most centers used eight fractions of 67.5 Gy prescribed to the $65-90 \%$ isodose surrounding the planning target volume (PTV) as their standard fractionation schedule for SBRT of peripherally and central lung tumors. About one quarter of the centers recommended three fractions of 12.5 Gy prescribed to an isodose line between $60-67 \%$. No center used single fraction regimens as standard. Center characteristics and treatment standards are summarized in Tables 1 and 2, respectively.

The following figures describe the tumor location and treatment recommendations for the nine cases. In the coronal (left-right), sagittal (anterior-posterior) and longitudinal (superior-inferior) axis the longest tumor diameters were measured. Furthermore, the maximum tumor diameter as well as the minimal distance to the carina, the mediastinum and the main bronchi are provided.
Table 2 Most common standard fractionation schedules for SBRT of lung tumors in general

\begin{tabular}{lllll}
\hline Fractions & Dose & $\begin{array}{l}\text { Isodose line } \\
\text { surrounding PTV }\end{array}$ & $\begin{array}{l}\text { Number } \\
\text { of centers }\end{array}$ & $\begin{array}{l}\text { Percentage } \\
\text { of centers }\end{array}$ \\
\hline 3 & 12.5 & $60-67 \%$ & 6 & $26 \%$ \\
5 & 7 & $65-80 \%$ & 2 & $9 \%$ \\
5 & 8 & $65 \%$ & 1 & $4 \%$ \\
6 & 8 & - & 1 & $4 \%$ \\
8 & 6 & $65-80 \%$ & 3 & $13 \%$ \\
8 & 7.5 & $70-90 \%$ & 9 & $39 \%$ \\
10 & 6 & $90 \%$ & 1 & $4 \%$ \\
\hline
\end{tabular}

\section{Case 1}

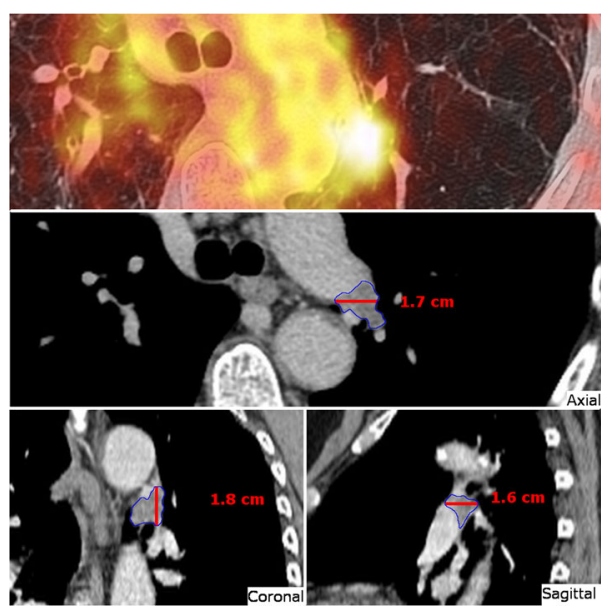

\begin{tabular}{|c|c|c|c|c|}
\hline \multicolumn{5}{|c|}{$\begin{array}{l}\text { Tumor location: Within left upper lobe, between left pulmonary artery } \\
\text { and left upper lobe bronchus }\end{array}$} \\
\hline $\begin{array}{l}\text { Shortest } \\
\text { distance } \\
\text { to } \\
\text { mediastinum } \\
{[\mathrm{cm}]}\end{array}$ & $\begin{array}{l}\text { Shortest } \\
\text { distance to } \\
\text { carina }[\mathrm{cm}]\end{array}$ & $\begin{array}{l}\text { Shortest } \\
\text { distance } \\
\text { to main } \\
\text { bronchus } \\
{[\mathrm{cm}]}\end{array}$ & $\begin{array}{l}\text { Size } \\
\text { (cor/sag/long) } \\
{[\mathrm{cm}]}\end{array}$ & $\begin{array}{l}\text { Maximum } \\
\text { diameter } \\
{[\mathrm{cm}]}\end{array}$ \\
\hline 0 & 3.8 & 0 & 1.7/1.6/1.8 & 1.9 \\
\hline $\begin{array}{l}\text { SBRT } \\
\text { accepted }\end{array}$ & $\begin{array}{l}\text { Most } \\
\text { frequently } \\
\text { used } \\
\text { fractionation }\end{array}$ & $\begin{array}{l}\text { Second most } \\
\text { frequently } \\
\text { used } \\
\text { fractionation }\end{array}$ & $\begin{array}{l}\text { Mean } \\
\text { number } \\
\text { of fractions }\end{array}$ & $\begin{array}{l}\text { Mean dose } \\
\text { per fraction } \\
{[G y]}\end{array}$ \\
\hline 21 (81\%) & $\begin{array}{l}8 \times 7.5 \text { Gy @ } \\
65-95 \%\end{array}$ & $\begin{array}{l}8 \times 6 \mathrm{~Gy} @ \\
65 \%\end{array}$ & 7.1 & 7.6 \\
\hline $\begin{array}{l}\text { SBRT } \\
\text { declined }\end{array}$ & \multicolumn{2}{|l|}{$\begin{array}{l}\text { Reasons to } \\
\text { decline SBRT }\end{array}$} & \multicolumn{2}{|c|}{$\begin{array}{l}\text { Most frequently } \\
\text { recommended alternative } \\
\text { treatment }\end{array}$} \\
\hline $5(19 \%)$ & \multicolumn{2}{|l|}{$\begin{array}{l}\text { Risk to central } \\
\text { airways }\end{array}$} & \multicolumn{2}{|c|}{$\begin{array}{l}\text { Conventionally } \\
\text { fractionated RT to } 70 G y\end{array}$} \\
\hline
\end{tabular}

Tumor characteristics of case 1. Abbreviations: cor coronal, sag sagittal, long longitudinal, SBRT Stereotactic body radiotherapy, $R T$ Radiotherapy, $R C T$ Radiochemotherapy 
Case 2

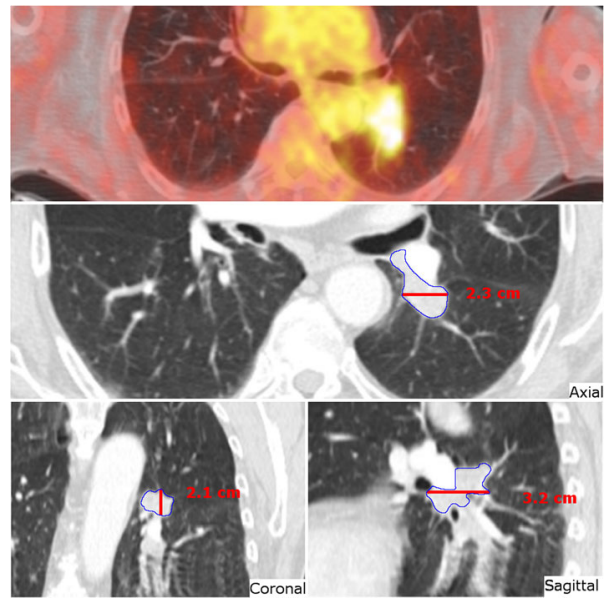

Tumor location: Within left lower lobe, adjacent to main bronchus

\begin{tabular}{|c|c|c|c|c|}
\hline $\begin{array}{l}\text { Shortest distance } \\
\text { to mediastinum } \\
{[\mathrm{cm}]}\end{array}$ & $\begin{array}{l}\text { Shortest } \\
\text { distance to } \\
\text { carina }[\mathrm{cm}]\end{array}$ & $\begin{array}{l}\text { Shortest } \\
\text { distance } \\
\text { to main } \\
\text { bronchus }[\mathrm{cm}]\end{array}$ & $\begin{array}{l}\text { Size } \\
\text { (cor/sag/ } \\
\text { long) [cm] }\end{array}$ & $\begin{array}{l}\text { Maximum } \\
\text { diameter } \\
{[\mathrm{cm}]}\end{array}$ \\
\hline 0 & 4.4 & 0 & 2.3/3.2/2.1 & 4.9 \\
\hline SBRT accepted & $\begin{array}{l}\text { Most } \\
\text { frequently } \\
\text { used } \\
\text { fractionation }\end{array}$ & $\begin{array}{l}\text { Second most } \\
\text { frequently } \\
\text { used } \\
\text { fractionation }\end{array}$ & $\begin{array}{l}\text { Mean } \\
\text { number } \\
\text { of fractions }\end{array}$ & $\begin{array}{l}\text { Mean } \\
\text { dose per } \\
\text { fraction } \\
{[G y]}\end{array}$ \\
\hline $0(77 \%)$ & $\begin{array}{l}8 \times 7.5 \text { Gy @ } \\
65-90 \%\end{array}$ & $\begin{array}{l}5 \times 7 \text { Gy @ } \\
65 \%\end{array}$ & 7.0 & 7.6 \\
\hline SBRT declined & \multicolumn{2}{|l|}{$\begin{array}{l}\text { Reasons to } \\
\text { decline SBRT }\end{array}$} & \multicolumn{2}{|c|}{$\begin{array}{l}\text { Most frequently } \\
\text { recommended } \\
\text { alternative treatment }\end{array}$} \\
\hline $6(23 \%)$ & \multicolumn{2}{|c|}{$\begin{array}{l}\text { Size, risk for left } \\
\text { main bronchus }\end{array}$} & \multicolumn{2}{|c|}{$\begin{array}{l}\text { Conventionally } \\
\text { fractionated RT } \\
\text { to 66-70Gy }\end{array}$} \\
\hline
\end{tabular}

Tumor characteristics of case 2. Abbreviations: cor coronal, sag sagittal, long longitudinal, SBRT Stereotactic body radiotherapy, $R T$ Radiotherapy, $R C T$ Radiochemotherapy

\section{Case 3}

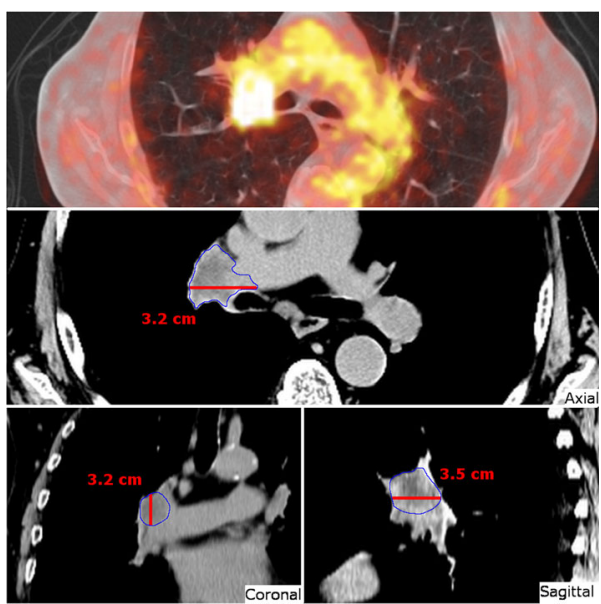

\begin{tabular}{|c|c|c|c|c|}
\hline \multicolumn{5}{|c|}{ Tumor location: Centrally located within the right hilus } \\
\hline $\begin{array}{l}\text { Shortest distance } \\
\text { to mediastinum } \\
{[\mathrm{cm}]}\end{array}$ & $\begin{array}{l}\text { Shortest } \\
\text { distance to } \\
\text { carina }[\mathrm{cm}]\end{array}$ & $\begin{array}{l}\text { Shortest } \\
\text { distance to } \\
\text { main bronchus } \\
{[\mathrm{cm}]}\end{array}$ & $\begin{array}{l}\text { Size (cor/ } \\
\text { sag/long) } \\
{[\mathrm{cm}]}\end{array}$ & $\begin{array}{l}\text { Maximum } \\
\text { diameter } \\
{[\mathrm{cm}]}\end{array}$ \\
\hline 0 & 2.5 & 0 & $3.2 / 3.5 / 3.2$ & 3.5 \\
\hline SBRT accepted & $\begin{array}{l}\text { Most } \\
\text { frequently } \\
\text { used } \\
\text { fractionation }\end{array}$ & $\begin{array}{l}\text { Second most } \\
\text { frequently used } \\
\text { fractionation }\end{array}$ & $\begin{array}{l}\text { Mean } \\
\text { number of } \\
\text { fractions }\end{array}$ & $\begin{array}{l}\text { Mean } \\
\text { dose per } \\
\text { fraction [Gy] }\end{array}$ \\
\hline $14(54 \%)$ & $\begin{array}{l}8 \times 7.5 \text { Gy } \\
@ 80-90 \%\end{array}$ & $\begin{array}{l}5 \times 8 \text { Gy } \\
@ 65 \%\end{array}$ & 7.5 & 6.3 \\
\hline SBRT declined & \multicolumn{2}{|c|}{ Reasons to decline SBRT } & \multicolumn{2}{|c|}{$\begin{array}{l}\text { Most frequently } \\
\text { recommended } \\
\text { alternative treatment }\end{array}$} \\
\hline $12(46 \%)$ & \multicolumn{2}{|c|}{ Risk for right main bronchus } & \multicolumn{2}{|c|}{$\begin{array}{l}\text { Conventionally } \\
\text { fractionated } \\
\text { RCT to } 66 \text { Gy }\end{array}$} \\
\hline
\end{tabular}

Tumor characteristics of case 3. Abbreviations: cor coronal, sag sagittal, long longitudinal, SBRT Stereotactic body radiotherapy, $R T$ Radiotherapy, $R C T$ Radiochemotherapy

\section{Case 4}

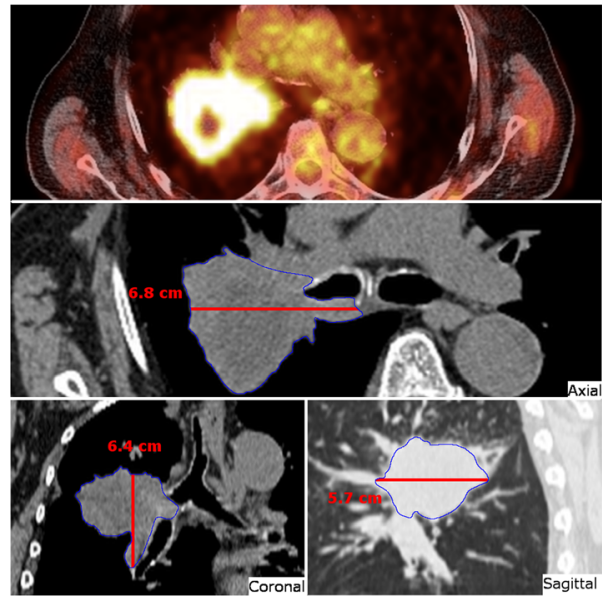

\begin{tabular}{|c|c|c|c|c|}
\hline \multicolumn{5}{|c|}{$\begin{array}{l}\text { Tumor location: large tumor reachin } \\
\text { bronchus and the main bronchus }\end{array}$} \\
\hline $\begin{array}{l}\text { Shortest } \\
\text { distance to } \\
\text { mediastinum [cm] }\end{array}$ & $\begin{array}{l}\text { Shortest } \\
\text { distance to } \\
\text { carina }[\mathrm{cm}]\end{array}$ & $\begin{array}{l}\text { Shortest distance } \\
\text { to main } \\
\text { bronchus }[\mathrm{cm}]\end{array}$ & $\begin{array}{l}\text { Size (cor/ } \\
\text { sag/long) } \\
{[\mathrm{cm}]}\end{array}$ & $\begin{array}{l}\text { Maximum } \\
\text { diameter } \\
{[\mathrm{cm}]}\end{array}$ \\
\hline 0 & 0 & 0 & $6.8 / 5.7 / 6.4$ & 7.6 \\
\hline SBRT accepted & $\begin{array}{l}\text { Most } \\
\text { frequently } \\
\text { used } \\
\text { fractionation }\end{array}$ & $\begin{array}{l}\text { Second most } \\
\text { frequently used } \\
\text { fractionation }\end{array}$ & $\begin{array}{l}\text { Mean } \\
\text { number } \\
\text { of } \\
\text { fractions }\end{array}$ & $\begin{array}{l}\text { Mean } \\
\text { dose per } \\
\text { fraction } \\
{[\text { Gy] }}\end{array}$ \\
\hline $1(4 \%)$ & $8 \times 5 \mathrm{~Gy}$ & - & - & - \\
\hline SBRT declined & \multicolumn{2}{|c|}{ Reasons to decline SBRT } & \multicolumn{2}{|c|}{$\begin{array}{l}\text { Most frequently } \\
\text { recommended } \\
\text { alternative treatment }\end{array}$} \\
\hline $25(96 \%)$ & \multicolumn{2}{|c|}{ Size, risk to central airways } & \multicolumn{2}{|c|}{$\begin{array}{l}\text { Conventionally } \\
\text { fractionated RCT to } \\
66 \mathrm{~Gy} \text {; Palliative RT } \\
30 \mathrm{~Gy} / 10 \mathrm{fr} \text {. }\end{array}$} \\
\hline
\end{tabular}


Tumor characteristics of case 4. Abbreviations: cor coronal, sag sagittal, long longitudinal, SBRT Stereotactic body radiotherapy, $R T$ Radiotherapy, $R C T$ Radiochemotherapy

\section{Case 5}

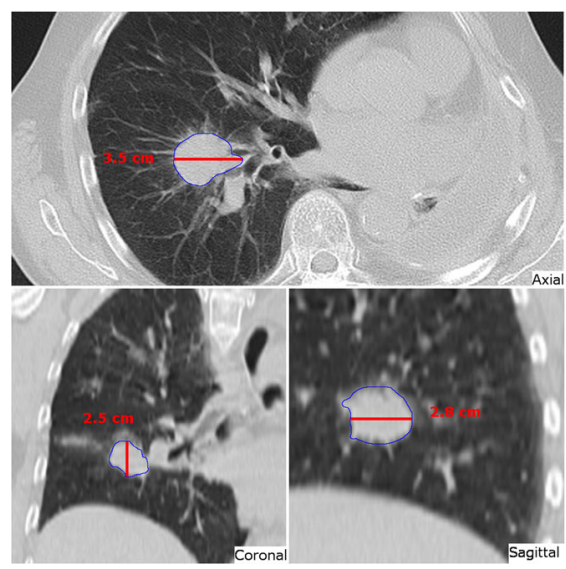

\begin{tabular}{|c|c|c|c|c|}
\hline \multicolumn{5}{|c|}{$\begin{array}{l}\text { Tumor location: centrally localized, next to right lower bronchus after } \\
\text { pneumonectomy of the left lung }\end{array}$} \\
\hline $\begin{array}{l}\text { Shortest } \\
\text { distance to } \\
\text { mediastinum } \\
{[\mathrm{cm}]}\end{array}$ & $\begin{array}{l}\text { Shortest } \\
\text { distance to } \\
\text { carina }[\mathrm{cm}]\end{array}$ & $\begin{array}{l}\text { Shortest distance } \\
\text { to main bronchus } \\
{[\mathrm{cm}]}\end{array}$ & $\begin{array}{l}\text { Size (cor/ } \\
\text { sag/long) } \\
{[\mathrm{cm}]}\end{array}$ & $\begin{array}{l}\text { Maximum } \\
\text { diameter } \\
{[\mathrm{cm}]}\end{array}$ \\
\hline 3.5 & 7.8 & 1.9 & $3.5 / 2.8 / 2.5$ & 3.5 \\
\hline SBRT accepted & $\begin{array}{l}\text { Most } \\
\text { frequently } \\
\text { used } \\
\text { fractionation }\end{array}$ & $\begin{array}{l}\text { Second most } \\
\text { frequently used } \\
\text { fractionation }\end{array}$ & $\begin{array}{l}\text { Mean } \\
\text { number of } \\
\text { fractions }\end{array}$ & $\begin{array}{l}\text { Mean dose } \\
\text { per fraction } \\
{[G y]}\end{array}$ \\
\hline $22(88 \%)$ & $\begin{array}{l}8 \times 7.5 \text { Gy @ } \\
65-90 \%\end{array}$ & 3 ×15 Gy @ 65\% & 6.0 & 9.4 \\
\hline SBRT declined & \multicolumn{2}{|c|}{ Reasons to decline SBRT } & \multicolumn{2}{|c|}{$\begin{array}{l}\text { Most frequently } \\
\text { recommended } \\
\text { alternative treatment }\end{array}$} \\
\hline $3(12 \%)$ & \multicolumn{2}{|c|}{ Localization too central } & \multicolumn{2}{|c|}{$\begin{array}{l}\text { Conventionally } \\
\text { fractionated RT }\end{array}$} \\
\hline
\end{tabular}

Tumor characteristics of case 5. Abbreviations: cor coronal, sag sagittal, long longitudinal, SBRT Stereotactic body radiotherapy, $R T$ Radiotherapy, $R C T$ Radiochemotherapy

\section{Case 6}

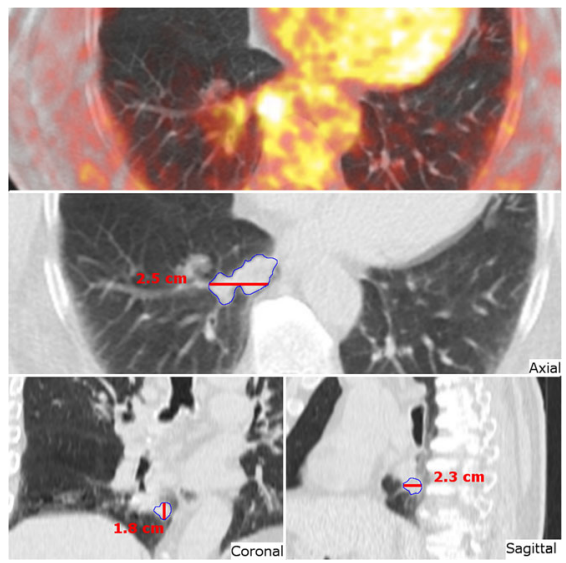

Tumor location: right lower lobe, adjacent to the esophagus

\begin{tabular}{|c|c|c|c|c|}
\hline $\begin{array}{l}\text { Shortest } \\
\text { distance to } \\
\text { mediastinum } \\
{[\mathrm{cm}]}\end{array}$ & $\begin{array}{l}\text { Shortest } \\
\text { distance to } \\
\text { carina }[\mathrm{cm}]\end{array}$ & $\begin{array}{l}\text { Shortest distance } \\
\text { to main } \\
\text { bronchus }[\mathrm{cm}]\end{array}$ & $\begin{array}{l}\text { Size (cor/ } \\
\text { sag/long) } \\
{[\mathrm{cm}]}\end{array}$ & $\begin{array}{l}\text { Maximum } \\
\text { diameter } \\
{[\mathrm{cm}]}\end{array}$ \\
\hline 0 & 7.5 & 2.2 & $2.5 / 2.3 / 1.8$ & 3.7 \\
\hline SBRT accepted & $\begin{array}{l}\text { Most } \\
\text { frequently } \\
\text { used } \\
\text { fractionation }\end{array}$ & $\begin{array}{l}\text { Second most } \\
\text { frequently used } \\
\text { fractionation }\end{array}$ & $\begin{array}{l}\text { Mean } \\
\text { number of } \\
\text { fractions }\end{array}$ & $\begin{array}{l}\text { Mean dose } \\
\text { per } \\
\text { fraction } \\
{[\text { Gy] }}\end{array}$ \\
\hline $2(88 \%)$ & $\begin{array}{l}8 \times 7.5 \text { Gy @ } \\
65-90 \%\end{array}$ & $\begin{array}{l}3 \times 13.5 / 12,5 \mathrm{~Gy} \\
\text { @ 65\% }\end{array}$ & 6.8 & 8.5 \\
\hline BRT declined & \multicolumn{2}{|c|}{ Reasons to decline SBRT } & \multicolumn{2}{|c|}{$\begin{array}{l}\text { Most frequently } \\
\text { recommended } \\
\text { alternative treatment }\end{array}$} \\
\hline (12\%) & \multicolumn{2}{|c|}{$\begin{array}{l}\text { Localization too central, risk to } \\
\text { esophagus }\end{array}$} & \multicolumn{2}{|c|}{$\begin{array}{l}\text { Surgery, conventionally } \\
\text { fractionated RT with } \\
\text { small margins }\end{array}$} \\
\hline
\end{tabular}

Tumor characteristics of case 6. Abbreviations: cor coronal, sag sagittal, long longitudinal, SBRT Stereotactic body radiotherapy, $R T$ Radiotherapy, $R C T$ Radiochemotherapy

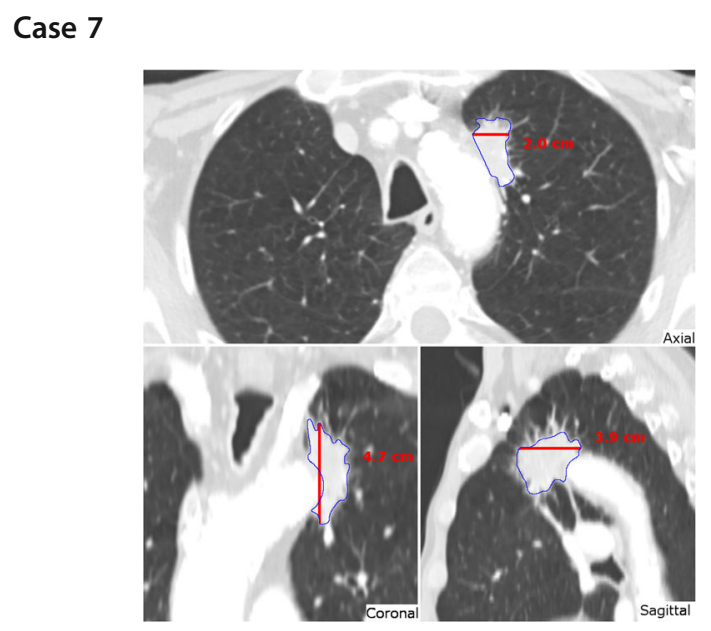

\begin{tabular}{|c|c|c|c|c|}
\hline \multicolumn{5}{|c|}{$\begin{array}{l}\text { Tumor location: Left upper lobe, Adjacent to the mediastinum at the height } \\
\text { of the aortic arch }\end{array}$} \\
\hline $\begin{array}{l}\text { Shortest } \\
\text { distance to } \\
\text { mediastinum } \\
{[\mathrm{cm}]}\end{array}$ & $\begin{array}{l}\text { Shortest } \\
\text { distance to } \\
\text { carina }[\mathrm{cm}]\end{array}$ & $\begin{array}{l}\text { Shortest distance } \\
\text { to main } \\
\text { bronchus }[\mathrm{cm}]\end{array}$ & $\begin{array}{l}\text { Size (cor/ } \\
\text { sag/long) } \\
{[\mathrm{cm}]}\end{array}$ & $\begin{array}{l}\text { Maximum } \\
\text { diameter } \\
{[\mathrm{cm}]}\end{array}$ \\
\hline 0 & 6.5 & 5 & 2.0/3.9/4.7 & 4.7 \\
\hline SBRT accepted & $\begin{array}{l}\text { Most } \\
\text { frequently } \\
\text { used } \\
\text { fractionation }\end{array}$ & $\begin{array}{l}\text { Second most } \\
\text { frequently used } \\
\text { fractionation }\end{array}$ & $\begin{array}{l}\text { Mean } \\
\text { number of } \\
\text { fractions }\end{array}$ & $\begin{array}{l}\text { Mean dose } \\
\text { per } \\
\text { fraction } \\
{[G y]}\end{array}$ \\
\hline $21(81 \%)$ & $\begin{array}{l}8 \text { x 7.5 Gy @ } \\
65-80 \%\end{array}$ & $\begin{array}{l}8 \text { x } 6 \text { Gy @ 65- } \\
80 \%\end{array}$ & 6.8 & 8.9 \\
\hline SBRT declined & \multicolumn{2}{|c|}{ Reasons to decline SBRT } & \multicolumn{2}{|c|}{$\begin{array}{l}\text { Most frequently } \\
\text { recommended } \\
\text { alternative treatment }\end{array}$} \\
\hline $5(19 \%)$ & \multicolumn{2}{|l|}{ Risk for aorta } & \multicolumn{2}{|c|}{$\begin{array}{l}\text { Conventionally } \\
\text { fractionated RT }+/- \\
\text { chemotherapy }\end{array}$} \\
\hline
\end{tabular}


Tumor characteristics of case 7. Abbreviations: cor coronal, sag sagittal, long longitudinal, SBRT Stereotactic body radiotherapy, $R T$ Radiotherapy, $R C T$ Radiochemotherapy

\section{Case 8}

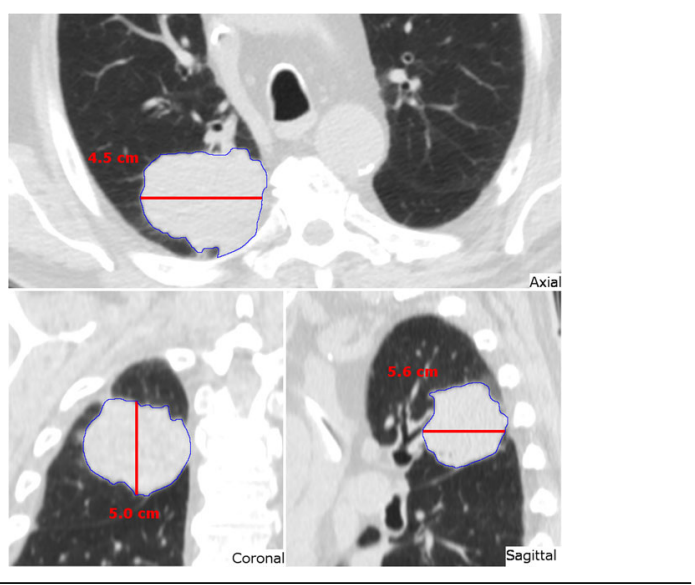

Tumor location: Large in size, right lower lobe, right after ramification of intermediate bronchi

\begin{tabular}{|c|c|c|c|c|}
\hline $\begin{array}{l}\text { Shortest } \\
\text { distance to } \\
\text { mediastinum } \\
{[\mathrm{cm}]}\end{array}$ & $\begin{array}{l}\text { Shortest } \\
\text { distance to } \\
\text { carina }[\mathrm{cm}]\end{array}$ & $\begin{array}{l}\text { Shortest distance } \\
\text { to main bronchus } \\
{[\mathrm{cm}]}\end{array}$ & $\begin{array}{l}\text { Size (cor/ } \\
\text { sag/long) } \\
{[\mathrm{cm}]}\end{array}$ & $\begin{array}{l}\text { Maximum } \\
\text { diameter } \\
{[\mathrm{cm}]}\end{array}$ \\
\hline 0 & 3.6 & 0.5 & $4.5 / 5.6 / 5.0$ & 5.7 \\
\hline SBRT accepted & $\begin{array}{l}\text { Most } \\
\text { frequently } \\
\text { used } \\
\text { fractionation }\end{array}$ & $\begin{array}{l}\text { Second most } \\
\text { frequently used } \\
\text { fractionation }\end{array}$ & $\begin{array}{l}\text { Mean } \\
\text { number of } \\
\text { fractions }\end{array}$ & $\begin{array}{l}\text { Mean dose } \\
\text { per fraction } \\
{[G y]}\end{array}$ \\
\hline $16(62 \%)$ & $\begin{array}{l}8 \text { x 7.5 Gy @ } \\
65-85 \%\end{array}$ & $\begin{array}{l}5 \text { x } 7 \text { Gy @ 60- } \\
65 \%\end{array}$ & 7.3 & 7.6 \\
\hline SBRT declined & \multicolumn{2}{|c|}{ Reasons to decline SBRT } & \multicolumn{2}{|c|}{$\begin{array}{l}\text { Most frequently } \\
\text { recommended } \\
\text { alternative treatment }\end{array}$} \\
\hline $10(38 \%)$ & \multicolumn{2}{|l|}{ Size } & \multicolumn{2}{|c|}{$\begin{array}{l}\text { Conventionally } \\
\text { fractionated RT, Palliative } \\
\text { hypofractionated RT }\end{array}$} \\
\hline
\end{tabular}

Tumor characteristics of case 8. Abbreviations: cor coronal, sag sagittal, long longitudinal, SBRT Stereotactic body radiotherapy, $R T$ Radiotherapy, $R C T$ Radiochemotherapy

\section{Case 9}

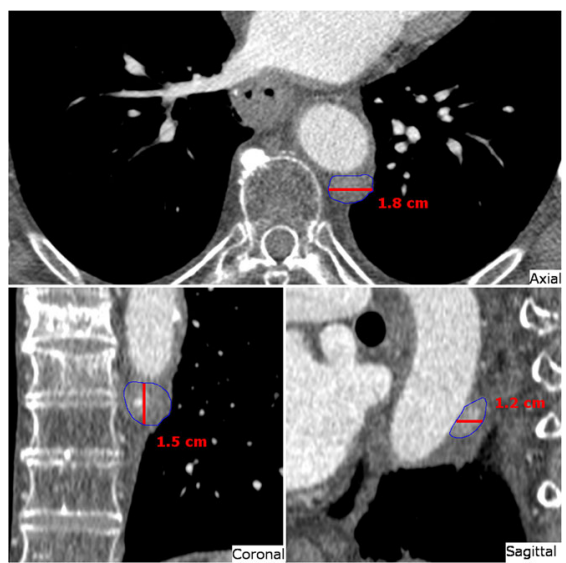

Tumor location: left lower lobe, adjacent to Aorta

\begin{tabular}{|c|c|c|c|c|}
\hline $\begin{array}{l}\text { Shortest } \\
\text { distance to } \\
\text { mediastinum } \\
{[\mathrm{cm}]}\end{array}$ & $\begin{array}{l}\text { Shortest } \\
\text { distance to } \\
\text { carina }[\mathrm{cm}]\end{array}$ & $\begin{array}{l}\text { Shortest } \\
\text { distance } \\
\text { to main } \\
\text { bronchus } \\
2[\mathrm{~cm}]\end{array}$ & $\begin{array}{l}\text { Size (cor/ } \\
\text { sag/long) } \\
{[\mathrm{cm}]}\end{array}$ & $\begin{array}{l}\text { Maximum } \\
\text { diameter } \\
{[\mathrm{cm}]}\end{array}$ \\
\hline 0 & 8.3 & 4.6 & $1.8 / 1.2 / 1.5$ & 2.1 \\
\hline $\begin{array}{l}\text { SBRT } \\
\text { accepted }\end{array}$ & $\begin{array}{l}\text { Most } \\
\text { frequently } \\
\text { used } \\
\text { fractionation }\end{array}$ & $\begin{array}{l}\text { Second most } \\
\text { frequently used } \\
\text { fractionation }\end{array}$ & $\begin{array}{l}\text { Mean } \\
\text { number } \\
\text { of } \\
\text { fractions }\end{array}$ & $\begin{array}{l}\text { Mean dose } \\
\text { per } \\
\text { fraction } \\
{[G y]}\end{array}$ \\
\hline $22(85 \%)$ & $\begin{array}{l}8 \times 7.5 \\
\text { Gy @ 65-90\% }\end{array}$ & $\begin{array}{l}3 \times 12,5 \text { Gy @ } \\
60-65 \%\end{array}$ & 6.6 & 8.7 \\
\hline $\begin{array}{l}\text { SBRT } \\
\text { declined }\end{array}$ & \multicolumn{2}{|l|}{$\begin{array}{l}\text { Reasons } \\
\text { to decline SBRT }\end{array}$} & \multicolumn{2}{|c|}{$\begin{array}{l}\text { Most frequently } \\
\text { recommended } \\
\text { alternative treatment }\end{array}$} \\
\hline$(15 \%)$ & \multicolumn{2}{|l|}{ Risk for aorta } & \multicolumn{2}{|c|}{ Palliative RT, surgery } \\
\hline
\end{tabular}

Tumor characteristics of case 9. Abbreviations: cor coronal, sag sagittal, long longitudinal, SBRT Stereotactic body radiotherapy, $R T$ Radiotherapy, $R C T$ Radiochemotherapy

\section{Discussion}

This survey demonstrates the current controversy regarding indications and contraindications for SBRT in central lung tumors, with a clear tendency towards the use of risk-adapted irradiation schedules. We categorized cases according to their level of acceptance (see Table 3) and identified three distinct decision criteria for SBRT: tumor size, exact location within the central zone and relation to the surrounding critical organs at risk, particularly the central airways.

Table 3 Cases grouped according to their level of acceptance

\begin{tabular}{|c|c|c|c|}
\hline Level of acceptance & High (>85\%) & Intermediate & Low $(<50 \%)$ \\
\hline Case Nr. & $5,6,9$ & $1,2,3,7,8$ & 4 \\
\hline $\begin{array}{l}\text { Most critical organ } \\
\text { at risk }\end{array}$ & $\begin{array}{l}\text { Main bronchus, } \\
\text { esophagus, aorta }\end{array}$ & $\begin{array}{l}\text { Main bronchus, } \\
\text { upper lobe } \\
\text { bronchus, aorta }\end{array}$ & $\begin{array}{l}\text { Main } \\
\text { bronchus }\end{array}$ \\
\hline $\begin{array}{l}\text { Maximal tumor } \\
\text { diameter } \\
\text { (range/median) }\end{array}$ & $\begin{array}{l}2.1-3.7 \mathrm{~cm} / \\
3.5 \mathrm{~cm}\end{array}$ & $\begin{array}{l}1.9-5.7 \mathrm{~cm} / \\
4.7 \mathrm{~cm}\end{array}$ & $7.6 \mathrm{~cm}$ \\
\hline $\begin{array}{l}\text { Minimal distance } \\
\text { to carina } \\
\text { (range/median) }\end{array}$ & $\begin{array}{l}7.5-8.3 \mathrm{~cm} / \\
7.8 \mathrm{~cm}\end{array}$ & $\begin{array}{l}2.5-6.5 \mathrm{~cm} / \\
3.8 \mathrm{~cm}\end{array}$ & $0 \mathrm{~cm}$ \\
\hline $\begin{array}{l}\text { Minimal distance } \\
\text { to main bronchus } \\
\text { (range/median) }\end{array}$ & $\begin{array}{l}1.9-4.6 \mathrm{~cm} / \\
2.2 \mathrm{~cm}\end{array}$ & $\begin{array}{l}0-0.5 \mathrm{~cm} / \\
0 \mathrm{~cm}\end{array}$ & $0 \mathrm{~cm}$ \\
\hline $\begin{array}{l}\text { Alternative } \\
\text { treatment }\end{array}$ & $\begin{array}{l}\text { RT } 70 \mathrm{~Gy} / \\
35 \mathrm{fr} .\end{array}$ & $\begin{array}{l}\text { RCT: } 66 \text { Gy/ } \\
33 \mathrm{fr} .\end{array}$ & $\begin{array}{l}\text { RT: } 30 \mathrm{~Gy} / 10 \\
\text { fr. or RCT: } \\
66 \mathrm{~Gy} / 33 \mathrm{fr} .\end{array}$ \\
\hline $\begin{array}{l}\text { Most frequently } \\
\text { stereotactic } \\
\text { fractionation }\end{array}$ & $60 \mathrm{~Gy} / 8 \mathrm{fr}$. & $60 \mathrm{~Gy} / 8 \mathrm{fr}$. & 40 Gy/8 fr. \\
\hline $\begin{array}{l}\text { Second most } \\
\text { frequently stereotactic } \\
\text { fractionation }\end{array}$ & $37,5-50 \mathrm{~Gy} / 3 \mathrm{fr}$. & $\begin{array}{l}35-40 \mathrm{~Gy} / 5 \mathrm{fr} . \\
48 \mathrm{~Gy} / 8 \mathrm{fr} .\end{array}$ & - \\
\hline
\end{tabular}


We observed a high agreement (>85\%) to perform SBRT despite central location for tumors with a maximal diameter of up to $4 \mathrm{~cm}$, a minimal distance to the main bronchi of about $2 \mathrm{~cm}$ and without infiltration of lobar bronchi.

These tumors were located within the central zone as defined in the RTOG 0813 trial, but mainly distally with regard to the original definition by Timmerman et al. $[12,18]$. The latter was used in the RTOG 0236 trial where the tumor was required to have a distance of at least $2 \mathrm{~cm}$ to the PBT, which was defined as the trachea, carina, main bronchi, and named major lobar bronchi up to their first bifurcation (i.e. upper and lower lobe bronchi, intermedius bronchus, lingular bronchus) $[15,16]$. RTOG 0813 included tumors within $2 \mathrm{~cm}$ to the PBT or touching the mediastinum [18]. Furthermore, case 5 showed that prior pneumonectomy was not considered as a contraindication even in centrally located tumors, offering these high-risk patients a potentially curative treatment option.

Lung tumors which had only an intermediate level of acceptance for SBRT (54-81\%) were located within the central zone as defined in RTOG 0813. SBRT was contraindicated by several centers due to close proximity to the central airways (case 1-3), particularly when the tumor was directly located at the hilus (case 2 ). These cases met the criteria of "ultra-central" tumors which can be defined in two ways: Either the gross tumor volume directly abutted the central airway or the PTV overlapped the trachea or main bronchi $[21,22]$.

In contrast, tumors which were immediately adjacent to the mediastinum and aorta had a higher acceptance for SBRT of $81-85 \%$ (case 3, 7 \& 9). A reduced level of acceptance was also observed for cases where the tumor diameter exceeded $4 \mathrm{~cm}$ (case 2, 4 and 8).

SBRT was rejected by almost all radiation oncologists in case 4: The tumor exceeded $6 \mathrm{~cm}$ in diameter in almost every dimension and extensively infiltrated the right major bronchus up to the carina.

Taken together, the most prominent contraindications for SBRT were proximity to the carina, possible infiltration of the central airways (tumor immediately adjacent to the main bronchus) and tumor size beyond $4 \mathrm{~cm}$. Each of the three criteria influenced the decision for or against SBRT independently, which is demonstrated in Fig. 1.

About half of the centers used schedules of eight fractions as their standard SBRT regimen for central lung tumors (Table 2), which was also the most frequently used regimen in the presented cases.

Sixty Gray in eight fractions is a common scheme for centrally located lung tumors propagated in particular by the group from VU University Medical Center Amsterdam/Netherlands [20, 23, 24]. The latest update of their series retrospectively compared 80 cases of central SBRT treated with eight fractions of 7.5 Gy (PTV overlapping a $2 \mathrm{~cm}$ expansion of the PBT according to RTOG 0813) to 252 cases of peripheral tumors treated with more escalated regimens [23]. There was no significant difference in overall survival between both groups and grade three pulmonary toxicity was observed in $6.4 \%$ of cases. In five cases the PTV overlapped the PBT, but only one patient developed a bronchial stricture and consecutively atelectasis. Although no grade four toxicity was described, six cases $(7.5 \%)$ were considered to have possible $(n=3)$ or likely $(n=3)$ treatment-related death. Low toxicity rates of this SBRT regimen were also seen by Taremi et al. and Guckenberger et al., where two of twenty-two patients with central tumors developed grade two/three toxicity after irradiation with $48 \mathrm{~Gy}$ in eight fractions prescribed to the $65 \%$ isodose $[25,26]$. As these data were not collected prospectively and patient cohorts show high pulmonary and cardiologic comorbidity, some toxicities may have escaped documentation. Furthermore, there may have been a low number of patients at risk for specific toxicities based on the dose distribution.

Five fraction regimens as second most common SBRT schedules were recommended in the intermediate acceptance group. More aggressive three fraction regimens were recommended only for tumors below $4 \mathrm{~cm}$ in size and distal of the PBT (high acceptance group). No center chose single-fraction radiotherapy.

It is important to mention that presented CT images were static. Additional information on tumor motion and

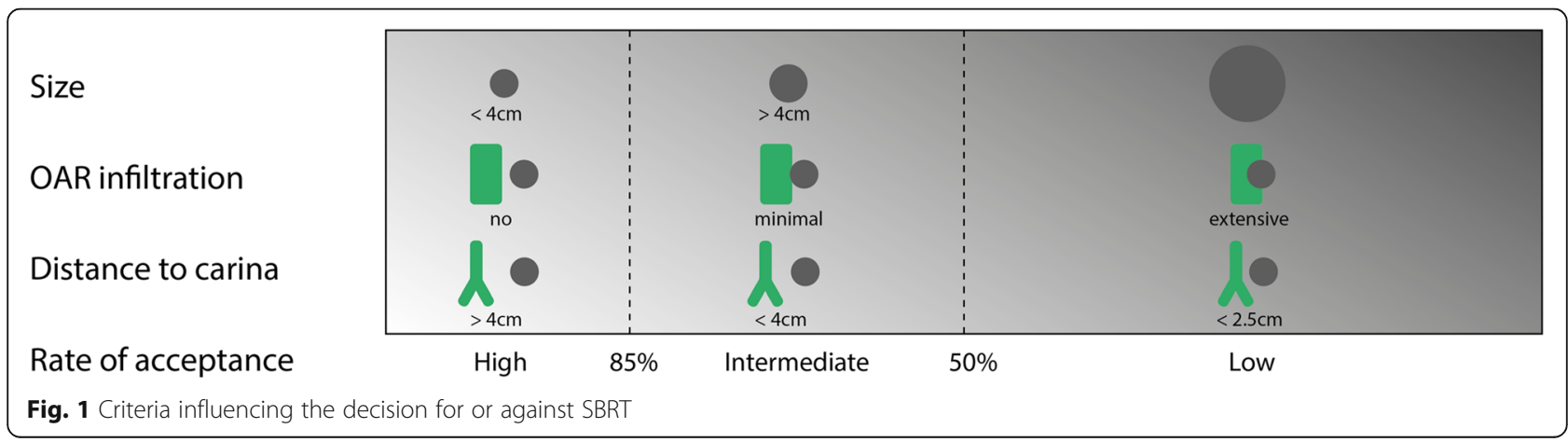


corresponding organs at risk certainly would have influenced the decision whether and how SBRT would have been carried out. Obviously, this survey does not include the results of patient-specific interdisciplinary case discussion and counseling. Furthermore, not all participants completed the whole questionnaire. Due to the small number of participants the statistical analysis is descriptive rather than inferential.

\section{Conclusion}

This survey showed a high level of acceptance of SBRT for selected cases with small centrally located tumors and minimal distance to the main bronchus of at least $2 \mathrm{~cm}$. Apart from tumor size, the exact location within the central zone and the relation to critical organs at risk were important decision criteria for or against SBRT. Despite contact or even infiltration of the mediastinum, SBRT was accepted in small tumors not abutting the main bronchi. Acceptance of SBRT clearly decreased if the maximal tumor diameter exceeded $4 \mathrm{~cm}$ and the minimal distance to the main bronchus was below $2 \mathrm{~cm}$. Secondly, we also observed a risk adaptation of SBRT fractionation within centrally located tumors, which was practiced in all participating institutions: higher risk tumors in terms of size and location were treated with a larger number of SBRT fractions and lower total SBRT doses. However, the variety of opinions on treatment of larger and more central tumors reflects the current controversy on this topic, which will hopefully be solved by running prospective clinical trials.

\section{Abbreviations \\ Cor: Coronal; CT: Computed tomography; DEGRO: Deutsche Gesellschaft für Radioonkologie e.V. (German society for radiation oncology); FDG- PET: Fluordeoxyglucose positron emission tomography; Fr.: Fractions; IASLC: International Association for the Study of Lung Cancer; Long: Longitudinal; NSCLC: Non-small cell lung cancer; PBT: Proximal bronchial tree; PTV: Planning target volume; RT: Radiation therapy; RTOG: Radiation Therapy Oncology Group; Sag: Sagittal; SBRT: Stereotactic body radiation therapy}

\section{Acknowledgements}

We would like to take this opportunity to thank all participating centers of the DEGRO working group on stereotactic radiotherapy. Furthermore, we gratefully thank Prof. J. Unkelbach and D. McDonnell, who carefully reviewed and substantially improved the manuscript.

\section{Availability of data and materials}

All data reported.

\section{Authors' contributions}

$J R$ analyzed the data, performed a literature review and drafted the manuscript. $\mathrm{CP}$ assisted in drafting of the manuscript. FS, FM, UN, NA provided cases and helped draft the manuscript. MG conceived the method, provided cases, assisted with data handling and helped draft the manuscript. All authors read and approved the final manuscript.

\section{Competing interests}

The authors declare that they have no competing interests.

Consent for publication

Not applicable.
Ethics approval and consent to participate

Not applicable.

\section{Author details}

'Department of Radiation Oncology, Universitässpital Zürich, Zürich, Switzerland. ${ }^{2}$ Heidelberg Institute of Radiation Oncology (HIRO), Heidelberg, Germany. ${ }^{3}$ Department of Radiation Oncology, Universitätsklinikum Würzburg, Würzburg, Germany. ${ }^{4}$ Department of Radiation Oncology, Universitätsklinikum Freiburg, Freiburg, Germany.

Received: 18 May 2016 Accepted: 21 November 2016

Published online: 03 December 2016

\section{References}

1. Ferlay J, Steliarova-Foucher E, Lortet-Tieulent J, Rosso S, Coebergh JWW, Comber $\mathrm{H}$, et al. Cancer incidence and mortality patterns in Europe: Estimates for 40 countries in 2012. Eur J Cancer. 2013;49:1374-403. doi:10. 1016/j.ejca.2012.12.027.

2. Jemal A, Bray F, Center MM, Ferlay J, Ward E, Forman D. Global cancer statistics. CA Cancer J Clin. 2011;61:69-90. doi:10.3322/caac.20107.

3. Ferlay J, Soerjomataram I, Ervik M, Dikshit R, Eser S, Mathers C, et al. GLOBOCAN 2012 v1.0, Lung Cancer Estimated Incidence, Mortality and Prevalence Worldwide in 2012 n.d.

4. Smith BD, Smith GL, Hurria A, Hortobagyi GN, Buchholz TA. Future of cancer incidence in the United States: burdens upon an aging, changing nation. J Clin Oncol. 2009:27:2758-65. doi:10.1200/JCO.2008.20.8983.

5. National Lung Screening Trial Research Team, Aberle DR, Adams AM, Berg CD, Black WC, Clapp JD, et al. Reduced lung-cancer mortality with low-dose computed tomographic screening. N Engl J Med. 2011;365:395-409. doi:10. 1056/NEJMoa1102873.

6. de Perrot M, Licker M, Reymond MA, Robert J, Spiliopoulos A. Influence of age on operative mortality and long-term survival after lung resection for bronchogenic carcinoma. Eur Respir J. 1999;14:419-22.

7. Chang JY, Senan S, Paul MA, Mehran RJ, Louie AV, Balter P, et al. Stereotactic ablative radiotherapy versus lobectomy for operable stage non-small-cell lung cancer: a pooled analysis of two randomised trials. Lancet Oncol. 2015;16:630-7. doi:10.1016/S1470-2045(15)70168-3.

8. Dahele M, Hatton M, Slotman B, Guckenberger M. Stereotactic body radiotherapy: A survey of contemporary practice in six selected European countries. Acta Oncol Stockh Swed 2015:1-5. doi:10.3109/0284186X.2014.1003961

9. Daly ME, Perks JR, Chen AM. Patterns-of-care for thoracic stereotactic body radiotherapy among practicing radiation oncologists in the United States. J Thorac Oncol Off Publ Int Assoc Study Lung Cancer. 2013;8:202-7. doi:10. 1097/JTO.0b013e318279155f.

10. Siegenthaler MP, Pisters KM, Merriman KW, Roth JA, Swisher SG, Walsh GL, et al. Preoperative chemotherapy for lung cancer does not increase surgical morbidity. Ann Thorac Surg. 2001;71:1105-12. doi:10.1016/S00034975(01)02406-7.

11. Saito M, Furukawa $K$, Miura T, Kato H. Evaluation of $T$ factor, surgical method, and prognostic factors in central type lung cancer. Jpn J Thorac Cardiovasc Surg Off Publ J.pn Assoc Thorac Surg Nihon Kyöbu Geka Gakkai Zasshi. 2002:50:413-7.

12. Timmerman R, McGarry R, Yiannoutsos C, Papiez L, Tudor K, DeLuca J, et al. Excessive toxicity when treating central tumors in a phase II study of stereotactic body radiation therapy for medically inoperable early-stage lung cancer. J Clin Oncol Off J Am Soc Clin Oncol. 2006;24:4833-9. doi:10. 1200/JCO.2006.07.5937.

13. Nestle U, Faivre-Finn C, DeRuysscher D, Hurkmans C, Guckenberger M, Belderbos J, et al. Stereotactic body radiotherapy (SBRT) in central non-small cell lung cancer (NSCLC): solid evidence or "no-go"? Radiother Oncol J Eur Soc Ther Radiol Oncol. 2013;109:178-9. doi:10.1016/j.radonc.2013.07.022.

14. Adebahr S, Collette S, Shash E, Lambrecht M, Le Pechoux C, Faivre-Finn C, et al. LungTech, an EORTC Phase II trial of stereotactic body radiotherapy for centrally located lung tumours: a clinical perspective. Br J Radiol. 2015;88: 20150036. doi:10.1259/bjr.20150036.

15. Timmerman RD, Paulus R, Galvin J, Michalski J, Straube W, Bradley J, et al. Stereotactic body radiation therapy for medically inoperable early-stage lung cancer patients: analysis of RTOG 0236. Int J Radiat Oncol. 2009;75:S3 doi:10.1016/j.jprobp.2009.07.033. 
16. Timmerman R, Paulus R, Galvin J, Michalski J, Straube W, Bradley J, et al. Stereotactic body radiation therapy for inoperable early stage lung cancer. JAMA J Am Med Assoc. 2010;303:1070-6. doi:10.1001/jama.2010.261.

17. Chang JY, Bezjak A, Mornex F, Advanced Radiation Technology Committee IASLC. Stereotactic ablative radiotherapy for centrally located early stage non-small-cell lung cancer: what we have learned. J Thorac Oncol Off Publ Int Assoc Study Lung Cancer. 2015;10:577-85. doi:10.1097/JTO. 0000000000000453 .

18. Bezjak A, Paulus R, Gaspar LE, Timmerman RD, Straube WL, Ryan WF, et al. Primary study endpoint analysis for NRG oncology/RTOG 0813 trial of Stereotactic Body Radiation Therapy (SBRT) for centrally located Non-Small Cell Lung Cancer (NSCLC). Int J Radiat Oncol Biol Phys. 2016;94:5-6. doi:10. 1016/.jijrobp.2015.10.040.

19. Nuyttens JJ, van der Voort van Zyp NC, Praag J, Aluwini S, van Klaveren RJ, Verhoef $C$, et al. Outcome of four-dimensional stereotactic radiotherapy for centrally located lung tumors. Radiother Oncol J Eur Soc Ther Radiol Oncol. 2012:102:383-7. doi:10.1016/.j.radonc.2011.12.023.

20. Haasbeek CJA, Lagerwaard FJ, Slotman BJ, Senan S. Outcomes of stereotactic ablative radiotherapy for centrally located early-stage lung cancer. J Thorac Oncol Off Publ Int Assoc Study Lung Cancer. 2011;6:2036-43. doi:10.1097/JTO.0b013e31822e71d8.

21. Chaudhuri AA, Tang C, Binkley MS, Jin M, Wynne JF, von Eyben R, et al. Stereotactic ablative radiotherapy (SABR) for treatment of central and ultra-central lung tumors. Lung Cancer Amst Neth. 2015;89:50-6. doi:10.1016/j.lungcan.2015.04.014.

22. Tekatli H, Haasbeek N, Dahele M, De Haan P, Verbakel W, Bongers E, et al. Outcomes of hypo-fractionated high dose radiotherapy in poor risk patients with "ultra-central" non-small cell lung cancer. J Thorac Oncol Off Publ Int Assoc Study Lung Cancer 2016. doi:10.1016/j.jtho.2016.03.008

23. Tekatli H, Senan S, Dahele M, Slotman BJ, Verbakel WFAR. Stereotactic ablative radiotherapy (SABR) for central lung tumors: Plan quality and longterm clinical outcomes. Radiother Oncol J Eur Soc Ther Radiol Oncol. 2015; 117:64-70. doi:10.1016/j.radonc.2015.09.028.

24. Lagerwaard FJ, Haasbeek CJA, Smit EF, Slotman BJ, Senan S. Outcomes of risk-adapted fractionated stereotactic radiotherapy for stage I non-small-cell lung cancer. Int J Radiat Oncol Biol Phys. 2008;70:685-92. doi:10.1016/j. ijrobp.2007.10.053.

25. Guckenberger M, Wulf J, Mueller G, Krieger T, Baier K, Gabor M, et al. Doseresponse relationship for image-guided stereotactic body radiotherapy of pulmonary tumors: relevance of 4D dose calculation. Int J Radiat Oncol Biol Phys. 2009;74:47-54. doi:10.1016/j.jijobp.2008.06.1939.

26. Taremi M, Hope A, Dahele M, Pearson S, Fung S, Purdie T, et al. Stereotactic Body Radiotherapy for Medically Inoperable Lung Cancer: Prospective, Single-Center Study of 108 Consecutive Patients. Int J Radiat Oncol. 2012:82: 967-73. doi:10.1016/j.jprobp.2010.12.039.

\section{Submit your next manuscript to BioMed Central and we will help you at every step:}

- We accept pre-submission inquiries

- Our selector tool helps you to find the most relevant journal

- We provide round the clock customer support

- Convenient online submission

- Thorough peer review

- Inclusion in PubMed and all major indexing services

- Maximum visibility for your research

Submit your manuscript at www biomedcentral.com/submit

CBiomed Central 